\title{
A Novel Ward-based Comprehensive Geriatric Assessment for Elderly Orthopedic Patients and Analysis of Risk Factors for Postoperative Complications
}

\section{Chao Kong}

Xuanwu Hospital of Capital Medical University

Yanhong Zhang

Xuanwu Hospital of Capital Medical University

Chaodong Wang

Xuanwu Hospital of Capital Medical University

Peng Wang

Xuanwu Hospital of Capital Medical University

Xiangyu Li

Xuanwu Hospital of Capital Medical University

\section{Wei Wang}

Xuanwu Hospital of Capital Medical University

\section{Yu Wang}

Xuanwu Hospital of Capital Medical University

Jianghua Shen

Xuanwu Hospital of Capital Medical University

Xiaoyi Ren

Xuanwu Hospital of Capital Medical University

Tianlong Wang

Xuanwu Hospital of Capital Medical University

Guoguang Zhao

Xuanwu Hospital of Capital Medical University

Shibao Lu ( $\sim$ shibaoluspine@sina.com )

Xuanwu Hospital of Capital Medical University

\section{Research Article}

Keywords: comprehensive geriatric assessment, elderly, risk factor, postoperative complication, orthopedic surgery 
Posted Date: March 8th, 2022

DOI: https://doi.org/10.21203/rs.3.rs-1349672/v1

License: (c) (i) This work is licensed under a Creative Commons Attribution 4.0 International License. Read Full License 


\section{Abstract}

Background: The effectiveness of comprehensive geriatric assessment (CGA) has been confirmed to reduce perioperative complications of geriatric patients. But there is no universally accepted standardization of CGA for orthopedic surgery. In this study, a novel CGA strategy was applied to evaluate the conditions of elderly patients undergoing orthopedic surgery from a broad view and to identify potential risk factors for postoperative complications.

Methods: A prospective cohort study was conducted from March 2019 to December 2020.The study enrolled patients for elective or confined orthopedic surgery if they were over the age of 75 years old. All patients were enrolled and treated with help from a multidisciplinary team. A structured CGA was conducted to identify high-risk elderly patients and to facilitate coordinated multidisciplinary team care by a geriatric team. The basic patient characteristics and CGA results were collected in addition to postoperative complication and mortality rates. Multivariate logistic regression analysis was used to identify risk factors for postoperative complications.

Results: A total of 214 patients with an age of $81.07 \pm 4.78$ (range, $75-100$ ) years were prospectively enrolled in this study. In total, 66 (30.8\%) complications were registered, including one death from myocardial infarction (mortality rate, $0.5 \%$ ). Poor ADL and IADL were accompanied by frailty, worse American Society of Anesthesiologists (ASA) score, visual analogue scale score, and nutritional status. Poor ADL was also associated with higher risks of falling, polypharmacy, and cardiac and respiration complications. Poor IADL was associated with higher risk of cardiac and respiration complications. Higher stroke risk was accompanied by higher risks of cardiac complications, delirium, and hemorrhage. Worse ASA scale was associated with worse ADL, IADL, and frailty, and higher delirium risk. Multivariate logistic regression analysis showed that spinal fusion (odds ratio [OR], $0.73 ; 95 \%$ confidence interval [CI], 0.65 to $0.83 ; p=0.0214)$, blood loss $(\mathrm{OR}, 1.68 ; 95 \% \mathrm{Cl}, 1.31$ to $2.01 ; \mathrm{p}=0.0168)$, ADL (severe dysfunction or worse) (OR, 1.45; $95 \% \mathrm{Cl}, 1.16$ to $1.81 ; \mathrm{p}=0.0413)$, IADL (serious dependence) $(\mathrm{OR}, 1.08 ; 95 \% \mathrm{Cl}, 1.33$ to $1.63 ; p=0.0436)$, renal function (CKD $\geq$ stage $3 a)(O R, 2.01 ; 95 \% \mathrm{Cl}, 1.54$ to $2.55 ; p=0.0133)$, and malnutrition(OR, $2.11 ; 95 \% \mathrm{Cl}, 1.74$ to $2.56 ; \mathrm{p}=0.0101)$ were independent risk factors for postoperative complications. All $\mathrm{P}<0.05$.

Conclusion: Spinal fusion, blood loss, ADL (severe dysfunction or worse), IADL (serious dependence), renal function (CKD $\geq$ stage $3 a$ ) and nutrition MNA (malnourished) were independent risk factors of postoperative complications following orthopaedic surgery in elderly patients.

\section{Background}

Based on statistics from United States Census Bureau [1], the world's population aged 65 years and over is predicted to reach 1.6 billion by the year 2050. In China, the population aged 65 years and over is projected to reach 500 million by the year 2050 according to the statistics of the sixth population census published by the National Bureau of Statistics [2]. With the worldwide increase in the aged population, a 
concurrent increase in the demand for surgical procedures and total expenditure for Medicare is also expected to rise [3-4]. As part of that overall surge in cost, orthopedic surgeons will face unique challenges in caring for this aging patient population.

Older individuals often have multiple chronic illnesses that limit their functional capacity and recovery [5]. Moreover, non-disease-associated problems, such as frailty, polypharmacy, decreased functional dependence, poor nutritional status, and cognitive decline may also complicate surgical procedures and postoperative recovery [6-7]. Despite the advances in surgical and anesthetic techniques, perioperative complications and mortality of geriatric patients are relatively higher [8-10]. In order to provide optimal care for the older surgical patient, a thorough assessment of the individual's health status and a plan of care during the perioperative period are essential to identify and address deficits.

Comprehensive geriatric assessment (CGA) is a multidimensional interdisciplinary diagnostic process to determine the medical, psychosocial, and functional capabilities of a frail elderly person in order to develop a coordinated and integrated plan to maximize overall health with aging [11-12]. As the gold standard for in-hospital care of older patients, the effectiveness of CGA has been confirmed by many studies [13-14]. Despite the benefits of current CGA, there are some limitations. First, there is no universally accepted standardization in the assessment. The focused domains and assessment tools can differ among studies [15-17]. Second, there is still uncertainty about identifying and targeting suitable patients who are most likely to benefit from CGA $[13,18]$.

Based on these considerations, the China's National Center for Clinical Medicine of Geriatric Diseases assembled a 23-member multidisciplinary panel from 10 domains. After one year, a novel CGA tool was established based on a focused and structured literature review. This new CGA tool consists of two parts: primary screening and multidisciplinary assessment. Multidisciplinary assessment included 21 specific forms covering activities of daily living, nutritional status, cardiac function, pulmonary function, renal function, hepatic function, frailty, cognition, depression, delirium, medications, and endocrine levels. The aim of this study was to evaluate the geriatric conditions of elderly patients undergoing orthopedic surgery from a broad view and to identify any potential risk factors for postoperative complications.

\section{Methods}

The protocol of this prospective study conducted from March 2019 to December 2020 was approved by the regional medical ethics committee and registered in Chinese clinical trial registry (ChiCTR1800020363). The inclusion criteria were: 1 ) aged $\geq 75$ years with an expected preoperative time of $\geq 2$ days, and 2) patients scheduled for elective or confined orthopedic surgery. Exclusion criteria were emergency surgery, day surgery, and surgery under local anesthesia. All patients provided informed consent before surgery.

\section{CGA}


All patients were enrolled and treated with help from a perioperative multidisciplinary team. Preparing patients for orthopedics surgery were seen in the orthopedics department for one-station interprofessional perioperative evaluation and care coordination. The multidisciplinary team consisting of geriatricians, nurse specialists, dietitians, pharmacists, and physicians from the departments of cardiology, anesthesiology, neurology, respiratory, nephrology, gastroenterology, and endocrinology. All members of the multidisciplinary team had more than 5 years of clinical experience. Preoperative multidisciplinary evaluation will be completed within 48 hours after the patient is admitted to hospital, the multidisciplinary team offered recommendations for risk-reducing strategies in the preoperative and postoperative periods by Aged Patient Perioperative Longitudinal Evaluation-Multidisciplinary DecisionMaking System (APPLE-MDT) [19]. To facilitate implementation of recommendations made before surgery, the multidisciplinary team collaborated with the surgical teams conduct preoperative optimization. In the postoperative period, the multidisciplinary team followed patients conduct evaluation complications and provide rehabilitation guidance before discharge.

\section{Data collection and outcome measures}

All clinical data were prospectively collected by a doctor and a nurse independent of this study. In this study, data consisted of two parts: primary screening and multidisciplinary assessment.

Primary screening included patient characteristics, routine blood tests, nutrition, electrolyte, blood glucose, coagulation index, and stress echocardiography, electrocardiogram, pulmonary function tests, transcranial color doppler, deep vein ultrasound of lower extremity. Multidisciplinary assessment was conducted with the use of specific assessment forms. Functional status was determined based on Activities of Daily Living (ADL) [20] and Instrumental Activities of Daily Living (IADL) [21]. Nutritional status was evaluated using the mini nutritional assessment (MNA) [22], pain using the visual analogue scale (VAS) [23], renal function using the chronic kidney disease (CKD) stage classification [24], liver function using the modified Child-Pugh classification [25], respiratory function using the Arozullah postoperative respiratory failure risk score [26], thrombus risk evaluation using the Caprin score [27], cardiac risk evaluation using Lee's revised cardiac risk index [28], anesthesia risk using the American Society of Anesthesiologists (ASA) scale [29], cognitive function using the Montreal Cognitive Assessment [30], anxiety using the Zung self-rating anxiety scale [31], depression evaluation using the Geriatric Depression Scale [32], and stroke risk using the Revised Framingham Stroke Risk Profile and the Essen Stroke Risk Score [33-34]. Perioperative risks of massive hemorrhage, falling, delirium, and polypharmacy were also evaluated.

Postoperative complications or mortality were defined as complications or death within 30 days after surgery [20].

\section{Statistical analysis}


Continuous variables are presented as the mean \pm standard deviation (SD) and analyzed with the independent-sample $t$-test. Categorical variables are presented as percentages and analyzed with the chisquare test. Spearman correlation analysis was used to analyze the association among different CGA domains. Multivariate logistic regression analysis was used to identify risk factors for postoperative complications. A probability $(p)$ value of $<0.05$ was considered statistically significant. All statistical analyses were performed using SPSS software (version 18.0; SPSS Inc., Chicago, IL, USA).

\section{Results}

From March 2019 to December 2020, eight patients were excluded during the primary screening process. A total of 214 patients with an average age of $81.07 \pm 4.78$ (range, 75-100) years were included for analysis. The demographic and clinical characteristics of all patients are listed in Table 1. Of all patients, 112 (52.3\%) underwent spinal fusion, 54 (25.2\%) had total knee replacement, and 48 (22.4\%) had total hip replacement. The most common comorbidity was hypertension, which was present preoperatively in $65.4 \%$ of the patients. Other common comorbidities were diabetes $(24.8 \%)$, coronary heart disease (21.5\%), cerebral infarction (14.3\%), arrhythmia (6.3\%), renal insufficiency (3.1\%), and chronic obstructive pulmonary disease (3.5\%). In addition, $26.6 \%$ of the patients had one comorbidity, $23.8 \%$ had two, $21 \%$ had three, $11.7 \%$ had more than three, and $16.8 \%$ had none. The mean number of comorbidities was 2.5 per patient (SD, 1.7). 
Table 1

Patient Demographic and Clinical Characteristics

\begin{tabular}{|c|c|c|}
\hline \multirow[b]{2}{*}{ Characteristic } & \multicolumn{2}{|c|}{ Patients ( $N=214)$} \\
\hline & No. & $\%$ \\
\hline Age, mean $\pm S D$, y & \multicolumn{2}{|c|}{$81.07 \pm 4.78$} \\
\hline Male sex & 72 & 33.6 \\
\hline $\mathrm{BMI}$, mean $\pm \mathrm{SD}$ & \multicolumn{2}{|c|}{$25.09 \pm 4.17$} \\
\hline Smoking (yes/no) & 22 & 10.3 \\
\hline Alcohol (yes/no) & 16 & 7.5 \\
\hline \multicolumn{3}{|l|}{ Comorbidities } \\
\hline 0 & 36 & 16.8 \\
\hline 1 & 56 & 26.2 \\
\hline 2 & 51 & 23.8 \\
\hline 3 & 45 & 21 \\
\hline$>3$ & 26 & 12.1 \\
\hline \multicolumn{3}{|l|}{ ASA scale } \\
\hline 2 & 39 & 18.2 \\
\hline 3 & 163 & 76.2 \\
\hline 4 & 12 & 5.6 \\
\hline \multicolumn{3}{|l|}{ Type of surgery } \\
\hline -Spinal fusion & 112 & 52.3 \\
\hline -Total knee replacement & 54 & 25.2 \\
\hline -Total hip replacement & 48 & 22.4 \\
\hline Surgical duration, mean $\pm S D$, min & \multicolumn{2}{|c|}{$151.50 \pm 86.74$} \\
\hline Blood loss, mean \pm SD, ml & \multicolumn{2}{|c|}{$285.53 \pm 316.34$} \\
\hline \multicolumn{3}{|l|}{ Abbreviations: } \\
\hline ASA, American Society of Anesthe & ss ind & ndard \\
\hline
\end{tabular}


One patient died of myocardial infarction 7 days after surgery, resulting in a mortality rate of $0.5 \%$. A total number of $66(30.8 \%)$ complications were registered. Implant-related complications are not discussed in this article. The most frequent postoperative complications were hypoproteinemia (albumin $\leq 30 \mathrm{~g} / \mathrm{L}$ ) $(9.8 \%)$, followed by wound infection (3.7\%), pneumonia (2.8\%), urinary tract infection $(2.3 \%)$, delirium $(1.9 \%)$, transient arrhythmia (1.4\%), pressure sores (1.4\%), cerebral infarction (1.4\%), myocardial infarction $(1.4 \%)$, ileus $(0.9 \%)$, gastrointestinal tract bleeding $(0.9 \%)$, respiratory failure $(0.9 \%)$, deep vein thrombosis $(0.5 \%)$, cardiac failure $(0.5 \%)$, and pulmonary embolus $(0.5 \%)$. The mean hospital stay was $13.45 \pm 7.46$ days.

Comparisons of demographic and clinical characteristics between patients with and without complications are shown in Table 2. Patients undergoing spinal fusion and those with more blood loss tended to have higher risks of postoperative complications $(p \leq 0.002)$. 
Table 2

Comparisons of Demographic and Clinical Characteristics between Patients with and without Complications

\begin{tabular}{|c|c|c|c|}
\hline Characteristic & $\begin{array}{l}\text { With complications } \\
(n=66)\end{array}$ & $\begin{array}{l}\text { Without complications } \\
(n=148)\end{array}$ & $p$ \\
\hline Mean age & $80.94 \pm 4.57$ & $81.14 \pm 5.14$ & 0.741 \\
\hline Male sex (n/\%) & $18(27.3 \%)$ & $54(36.5 \%)$ & 0.188 \\
\hline BMI & $24.83 \pm 4.48$ & $25.21 \pm 3.97$ & 0.684 \\
\hline Smoking (yes/\%) & $10(15.2 \%)$ & $12(8.1 \%)$ & 0.117 \\
\hline Alcohol (yes/\%) & $7(10.6 \%)$ & $9(6.1 \%)$ & 0.245 \\
\hline Comorbidities & & & 0.360 \\
\hline no & 10 & 26 & \\
\hline 1 & 13 & 43 & \\
\hline 2 & 15 & 36 & \\
\hline 3 & 17 & 28 & \\
\hline$>3$ & 11 & 15 & \\
\hline ASA scale & & & 0.289 \\
\hline 2 & 13 & 26 & \\
\hline 3 & 47 & 116 & \\
\hline 4 & 6 & 6 & \\
\hline Type of surgery & & & $<0.001$ * \\
\hline -Spinal fusion & 44 & 68 & \\
\hline -Total knee replacement & 5 & 49 & \\
\hline -Total hip replacement & 17 & 31 & \\
\hline Surgical duration (min) & $160.46 \pm 91.62$ & $147.72 \pm 81.60$ & 0.475 \\
\hline Blood loss (ml) & $438.65 \pm 479.14$ & $217.24 \pm 252.75$ & $0.002^{*}$ \\
\hline \multicolumn{4}{|c|}{ Abbreviations: ASA, American Society of Anesthesiologists; BMI, body mass index. } \\
\hline
\end{tabular}




\section{Preoperative CGA results}

All patients received a thorough CGA with multidisciplinary assessment completed within $48 \mathrm{~h}$. About $63.1 \%$ of all patients had moderate or severe pain before surgery, $28.5 \%$ had severe or extremely severe dysfunction and were seriously dependent before surgery, $66.4 \%$ were frail, and $14 \%$ were malnourished before surgery. Most patients had satisfactory perioperative blood glucose control (89.25\%) and normal thyroid function (86.92\%). Renal function was stage 3a or worse in 101 (47.2\%) patients, while 93.93\% had normal liver function, as determined with the modified Child-Pugh classification.

Overall, 75 patients (35.05\%) were at high risk for cardiac complications, 196 (91.59\%) were at extremely high risk for thrombus, 176 patients (82.24\%) had a high risk of massive hemorrhage. Respiration risk distributions were $0.5 \%$ (49 patients, $22.89 \%$ ), 1.8\% (88 patients, $41.12 \%$ ), $4.2 \%$ (68 patients, $31.78 \%$ ), and $10.1 \%$ (9 patients, $4.21 \%$ ). In addition, the risk of stroke was high in 42 patients (19.63\%), delirium in 16 (7.48\%), falling in 11 (5.14\%), and polypharmacy in 160 (74.77\%).

Mild cognitive impairment was identified in 113 patients (52.8\%). Five patients (2.34\%) had anxiety and 5 (2.34\%) had depression before surgery. The distribution of perioperative ASA scale was: grade II (42 patients, 19.63\%), grade III (156 patients, $72.9 \%$ ), and grade IV (16 patients, $7.47 \%$ ).

Spearman correlation analysis was used to analyze the association among different evaluation items. Poor ADL and IADL were accompanied by frailty and worse ASA, VAS, and nutritional status. Poor ADL was also associated with higher risks of falling, polypharmacy, cardiac complications, and respiration complications. Poor IADL was associated with higher risks of cardiac and respiration complications. Higher stroke risk was accompanied with higher risks of cardiac complications, delirium, and hemorrhage. A poor ASA score was associated with worse ADL, IADL, frailty, and higher delirium risk. Other associations of CGA results are shown in a correlation matrix presented in Table 3.

\section{Univariate Analysis of Potential Risk Factors Related to Complications}

The associations between postoperative complications and domains of the CGA are listed in Table 4 (other domains are listed in supplementary file). Univariate analysis showed that six domains were significantly associated with postoperative complications. Patients with dependent ADL, dependent IADL, worse renal function, worse nutritional status, cognitive impairment, and low ASA scores tended to have higher risk of postoperative complications $(p \leq 0.0442)$. 
Table 4

Univariate Analysis of Preoperative CGA Related to Postoperative Complications

Number (with any complication, \%)

$p^{a}$

ADL

$0.0296^{b}$

independent

$21(19.0 \%)$

mild dysfunction

$58(23.7 \%)$

moderate dysfunction

$75(28.4 \%)$

severe dysfunction

$45(39.5 \%)$

extremely severe dysfunction

$15(58.8 \%)$

IADL

$0.0354^{b}$

normal

$34(17.6 \%)$

mild dependence

$39(25.6 \%)$

moderate dependence

$80(28.8 \%)$

serious dependence

$61(44.3 \%)$

Nutrition (MNA)

$0.0442^{b}$

normal

114(23.7\%)

at risk of malnutrition

70(37.1\%)

malnourished

$30(43.3 \%)$

\section{Cognitive impairment}

$0.0052^{b}$

no impairment

101(19.8\%)

mild impairment

113(40.7\%)

Renal function

$0.0134^{b}$

normal

$7(0 \%)$

stage 1

$22(22.7 \%)$

stage 2

$84(23.8 \%)$

stage $3 a$

$69(44.9 \%)$

Abbreviations: ADL, Activities of Daily Living; ASA, American Society of Anesthesiologists; IADL, Instrumental Activities of Daily Living; MNA, Mini nutritional assessment; VAS, visual analogue scale; .

${ }^{a}$ Kruskal-Wallis test.

bSignificant differences among groups. 


\begin{tabular}{|c|c|c|}
\hline & Number (with any complication, \%) & $p^{a}$ \\
\hline stage $3 b$ & $26(38.5 \%)$ & \\
\hline stage 4 & $4(0 \%)$ & \\
\hline stage 5 & $2(0 \%)$ & \\
\hline ASA score & & $0.0376^{b}$ \\
\hline grade II & $39(16.7 \%)$ & \\
\hline grade III & $163(35.3 \%)$ & \\
\hline grade IV & $12(25.0 \%)$ & \\
\hline \multicolumn{3}{|c|}{$\begin{array}{l}\text { Abbreviations: ADL, Activities of Daily Living; ASA, American Society of Anesthesiologists; IADL, } \\
\text { Instrumental Activities of Daily Living; MNA, Mini nutritional assessment; VAS, visual analogue scale; }\end{array}$} \\
\hline \multicolumn{3}{|c|}{${ }^{a}$ Kruskal-Wallis test. } \\
\hline bSignifica & & \\
\hline
\end{tabular}

\section{Multivariate Analysis of Significant Univariate Factors}

Eight potential risk factors (i.e., type of surgery, blood loss, ADL, IADL, nutritional status, renal function, ASA score, and cognitive impairment) were included for logistic regression analysis. Multivariate analysis showed that spinal fusion (odds ratio [OR], $0.73 ; 95 \%$ confidence interval [Cl], 0.65 to $0.83 ; p=0.0214$ ), blood loss (OR, $1.68 ; 95 \% \mathrm{Cl}, 1.31$ to $2.01 ; p=0.0168)$, ADL (severe dysfunction or worse) $(\mathrm{OR}, 1.45 ; 95 \%$ $\mathrm{Cl}, 1.16$ to $1.81 ; p=0.0413)$, IADL (serious dependence) (OR, 1.08; $95 \% \mathrm{Cl}, 1.33$ to $1.63 ; p=0.0436$ ), renal function (CKD $\geq$ stage $3 a)(\mathrm{OR}, 2.01 ; 95 \% \mathrm{Cl}, 1.54$ to $2.55 ; p=0.0133)$, and malnutrition (MNA) (OR, 2.11; $95 \% \mathrm{Cl}, 1.74$ to $2.56 ; p=0.0101$ ) were independent risk factors for postoperative complications (Fig. 1 ).

\section{Discussion}

As the population continues to age, the demand for surgical services perioperative care will significantly increase. Surgical decisions for elderly, frailer patients require not only fitness for the procedure itself, but also the consideration of other factors, such as comorbidities, disability, cognitive impairment, and nutritional status. Many studies have described proactive and coordinated multidisciplinary assessment for elderly patients undergoing surgery [35-37]. However, these studies employed limited evaluation domains and few have focused on elective orthopedic surgery, especially spine surgery.

In this study, a novel CGA preoperative assessment was adopted that incorporated 21 specific forms from 10 domains. A thorough manifestation of geriatric conditions was shown in this study. Most patients in this cohort study underwent elective surgery with the aim of improving the quality of life, which explains 
the high prevalence of severe pain and dependence in daily life. One characteristic of the aging body is a reduction of physiological reserve, or frailty. More than $66 \%$ of all patients in this study were frail, which was higher than those reported in Fried's study [38]. Their patients were younger with less pain and dysfunction, which may have influenced the results. Kaiser et al. [39] reported that the prevalence of malnutrition among the elderly was $22.8 \%$, with considerable differences between the settings studied (rehabilitation, 50.5\%; hospital, 38.7\%; nursing home, $13.8 \%$; community, $5.8 \%$ ). The proportion of malnutrition in this study was merely $14 \%$, much lower than in the general elderly population. As a possible explanation, most patients who successfully underwent elective orthopedic surgery had a higher average life expectancy before surgery and were more likely to eat and live healthy. The prevalence of cognitive impairment among those aged $\geq 71$ years in the United States in 2002 was reportedly $22.3 \%$ [40]. In China, an estimated $20 \%$ of the population older than 55 years reportedly have cognitive impairment [41]. The prevalence of cognitive impairment reported in this study was higher than in a population of healthy older adults, but lower than elderly patients undergoing surgery for spinal deformities [42].

Though numerous studies have described preoperative CGA results, few have examined the inner relationships among different evaluation items. In this study, we established a correlation matrix of CGA results using Spearman correlation analysis. As shown in Table 4, poor ADL and IADL were accompanied by frailty and worse ASA, VAS, and nutritional status. Higher stroke risk was accompanied with higher risks for cardiac complications, delirium, and hemorrhage. Worse ASA scores were associated with worse $A D L$ and IADL, as well as frailty and higher delirium risk. Moreover, the cumulative effect of evaluation results should be interpreted with caution. Makary et al. [43] found that frailty was a better predictor of outcomes when combined with other evaluation forms. So, before surgery or intervention management, it is necessary to make sure that all preoperative evaluation results are handled as a whole.

The overall mortality and complication rates in this study were $0.5 \%$ and $30.8 \%$, respectively. The mortality rate was relatively lower, while the complication rate was consistent with other similar studies. In a retrospective study of consecutive elderly patients undergoing noncardiac surgery, the postoperative mortality and adverse outcomes rates were $4.6 \%$ and $25 \%$, respectively [6]. In the Surgical Quality Improvement Program database of the American College of Surgeons National, including standardized preoperative, intraoperative, and 30-day postoperative data points, the morbidity and mortality rates of a total of 7,696 surgical procedures were $28 \%$ and $2.3 \%$, respectively [7].

The purpose of preoperative assessment is to identify patients with an elevated risk for poor surgical outcomes. Age, by itself, does not increase surgical risk; rather, the increased prevalence of chronic disease and the deterioration of the organ function associated with aging might increase the risk of postoperative complications. In this study, four CGA domains (i.e., ADL, IADL, renal function, and nutritional status) were significantly associated with increased risks of postoperative complications.

Functional status is known to be a significant predictor of postoperative outcomes. Arozullah et al. [44] found that poor functional status was a risk factor for postoperative pneumonia. In a prospective case- 
controlled study of 509 patients, Chen et al. [45] found that the need for assistance with three or more $A D L s$ is an independent risk factor for surgical infection. In this study, ADL (with severe dysfunction or worse) and IADL (serious dependence) were significant independent risk factors for postoperative complications. Poor functional status has also been reported to be associated with nursing home placement [46]. Since assessment of functional status can be performed even in a busy preoperative setting to obtain valuable information, it should be routinely included in the preoperative assessment. Nutritional status is extremely important for elderly patients undergoing surgery. Multiple scoring systems have been developed to assess nutritional status, which include subjective global assessment, the Nutritional Risk Index, the MNA-short form, and the Maastricht Index. The European Society for Clinical Nutrition and Metabolism advocates the use of the MNA as a screening tool, while members of the American Society for Parenteral and Enteral Nutrition do not recommend any one screening tool over another [47]. No matter which tool is chosen, malnutrition is believed to be associated with postoperative complications, especially severe infection [48-49]. As reported in previous studies, the severity of renal disease is associated with higher rates of postoperative complications, especially those patients with end-stage renal disease requiring hemodialysis [50-51]. The use of a large nationwide database to compare outcomes between renal transplant and dialysis patients with diabetes after total hip arthroplasty found that renal dialysis was associated with increased risk of complication within 30 days after surgery [52]. Lieberman et al. [53] reported a deep infection rate of $19 \%$ and revision rate of $38 \%$ among dialysis-dependent patients as opposed to rates of $3 \%$ and $10 \%$ in patients with a renal transplant in a retrospective study. In this study, renal function with CKD $\geq$ stage $3 a$ was found to be independently associated with increased risk of postoperative complications.

There were several limitations to this study. First, the number of patients was relatively small, which may have caused statistical bias. Second, emergency surgery was excluded because of the lack of time to perform CGA in clinical practice. However, almost all spinal surgeries and total knee replacements were elective procedures. Third, because of the small number of patients, we were unable to establish a connection between specific postoperative complications and the preoperative CGA results.

\section{Conclusion}

The results of this study will allow orthopedic surgeons to better counsel their patients on the risks of postoperative complication. Spinal fusion, blood loss, ADL (severe dysfunction or worse), IADL (serious dependence), renal function (CKD $\geq$ stage $3 a$ ), and nutritional status (MNA, malnourished) were independent risk factors for postoperative complications in elderly patients following orthopedic surgery.

\section{Declarations}

\section{Ethics approval and consent to participate}

This study was reviewed and approved by the regional medical ethics committee and registered in Chinese clinical trial registry (ChiCTR1800020363). Informed consent was provided by all participating 
individuals.

\section{Consent for publication}

Not applicable

\section{Availability of data and material}

All data generated or analysed during this study are included in this published article [and its supplementary information files].

\section{Competing interests}

The authors declare no financial and non-financial competing interests.

\section{Funding}

This study was funded by the Beijing Municipal Medical Science Institute-Public Welfare Development Reform Pilot Project (Capital Medical Research No. 2019-2) and National Key Research and Development Program of China (No.2020YFC2004900).

\section{Author's contributions}

$\mathrm{KC}$ and $\mathrm{ZYH}$ analyzed and interpreted the patient data and wrote the first draft of manuscript. WP, LXY, WW and WY analyzed the data and did the follow-ups. SJH and RXY performed the CGA assessment and collected the data. WCD performed the statistical analysis and revised the manuscript. WTL, ZGG and LSB designed and directed the project and wrote the final manuscript. All authors have read and approved the manuscript.

\section{Acknowledgements}

We acknowledge the department of anesthesiology, nutriology, pharmacy, cardiology, neurology, and respiratory for MDT assessment. Especially multidisciplinary team member of Xiangyao Sun, Junzhe Ding, Wei Xiao, Long Fan, Yachao Xu, Xuexin Feng, Na Xu, Fei Lan, Lei Zhao.

\section{Ethics approval}

This study was reviewed and approved by the regional medical ethics committee and registered in Chinese clinical trial registry (ChiCTR1800020363).

\section{Human and animal rights}

This study has been performed in accordance with the ethical standards as laid down in the 1964 Declaration of Helsinki and its later amendments or comparable ethical standards. No animals have been involved. 


\section{References}

1. United States Census Bureau [homepage on the Internet]. U.S. Population Aging Slower than Other Countries, Census Bureau Reports. 2016. Available from: http://www.census.gov/newsroom/pressreleases/2016/ cb16-54.html?intcmp sd|pop|03282016. Accessed April 26, 2018.

2. Yanmei C, Zifeng L, Xiande L, Yixiang H. The Aging Trend of Chinese Population and the Prediction of Aging Population in 2015-2050. Chinese Journal of Social Medicine, 2018, 35(5): 480-483.

3. Dall TM, Gallo PD, Chakrabarti R, West T, Semilla AP, Storm MV. An aging population and growing disease burden will require a large and specialized health care workforce by 2025 . Health Aff (Millwood). 2013;32(11):2013-2020.

4. Etzioni DA, Liu JH, Maggard MA, Ko CY. The aging population and its impact on the surgery workforce. Ann Surg. 2003;238(2): 170-177.

5. Balducci L. Epidemiology of cancer and aging. J Oncol Manag 2005;14:47-50.

6. Liu LL, Leung JM. Predicting adverse postoperative outcomes in patients aged 80 years or older. J Am Geriatr Soc 2000;48:405-12.

7. Turrentine FE, Wang H, Simpson VB, et al. Surgical risk factors, morbidity, and mortality in older patients. J Am Coll Surg 2006;203: 865-77.

8. Deiner S, Westlake B, Dutton RP. Patterns of surgical care and complications in older adults. J Am Geriatr Soc 2014;62:829-35.

9. Vonlanthen R, Slankamenac K, Breitenstein S, et al. The impact of complications on costs of major surgical procedures. Ann Surg. 2011;254:907e913.

10. Lees MC, Merani S, Tauh K, Khadaroo RG. Perioperative factors predicting poor outcome in elderly patients following emergency general surgery: a multivariate regression analysis. Can J Surg. 2015;58:312e317.

11. Ellis G, Marshall T, Ritchie C. Comprehensive geriatric assessment in the emergency department. Clin Interv Aging. 2014 Nov 24;9:2033-43.

12. Parker SG, McLeod A, McCue P, Phelps K, Bardsley M, Roberts HC, Conroy SP. New horizons in comprehensive geriatric assessment. Age Ageing. 2017 Sep 1;46(5):713-721.

13. Parker SG, McCue P, Phelps K, McCleod A, Arora S, Nockels K, Kennedy S, Roberts H, Conroy S. What is Comprehensive Geriatric Assessment (CGA)? An umbrella review. Age Ageing. 2018 Jan 1;47(1):149-155.

14. Ellis G, Whitehead MA, Robinson D, O'Neill D, Langhorne P. Comprehensive geriatric assessment for older adults admitted to hospital: meta-analysis of randomised controlled trials. BMJ. 2011 Oct 27;343:d6553.

15. Kim KI, Park KH, Koo KH, Han HS, Kim CH. Comprehensive geriatric assessment can predict postoperative morbidity and mortality in elderly patients undergoing elective surgery. Arch Gerontol Geriatr. 2013, 56(3):507-12. 
16. Abete P, Cherubini A, Di Bari M, Vigorito C, Viviani G, Marchionni N, D'Ambrosio D, Golino A, Serra R, Zampi E, Bracali I, Mello A, Vitelli A, Rengo G, Cacciatore F, Rengo F. Does comprehensive geriatric assessment improve the estimate of surgical risk in elderly patients? An Italian multicenter observational study. Am J Surg. 2016 Jan;211(1):76-83.e2.

17. Braude P, Goodman A, Elias T, Babic-IIIman G, Challacombe B, Harari D, Dhesi JK. Evaluation and establishment of a ward-based geriatric liaison service for older urological surgical patients: Proactive care of Older People undergoing Surgery (POPS)-Urology. BJU Int. 2017 Jul;120(1):123129.

18. Ellis G, Langhorne P. Comprehensive geriatric assessment for older hospital patients. Br Med Bull 2004;71:45-59.

19. Zhang Y, Ma L, Wang T, Xiao W, Lu S, Kong C, Wang C, Li X, Li Y, Yin C, Yan S, Li Y, Yang K, Chan P, Group A-MSW (2021) Protocol for evaluation of perioperative risk in patients aged over 75 years: Aged Patient Perioperative Longitudinal Evaluation-Multidisciplinary Trial (APPLE-MDT study). BMC Geriatr 21:14.

20. KatzS, FordAB, MoskowitzRW, et al. Studies of illness in the aged: The index of ADL-A standardized measure of biological and psychosocial function. JAMA 185:914-919, 1963.

21. Lawton MP, Brody EM: Assessment of older people: Self-maintaining and instrumental activities of daily living. Gerontologist 9:179-186, 1969

22. Cohendy R, Rubenstein LZ, Eledjam JJ. The Mini Nutritional Assessment-Short Form for preoperative nutritional evaluation of elderly patients. Aging (Milano). 2001, 13(4):293-7.

23. Heller GZ, Manuguerra M, Chow R. How to analyze the Visual Analogue Scale: Myths, truths and clinical relevance. Scand J Pain. 2016, 13:67-75.

24. Hallan SI, Orth SR. The conundrum of chronic kidney disease classification and end-stage renal risk prediction in the elderly-what is the right approach? Nephron Clin Pract. 2010;116(4):c307-16.

25. Kumbasar A, Navdar M, Ataoglu E, Uzunhasan I, Ergen K, Poturoglu S, Basinoglu F, Yilmaz F, Yenigun M, Sar F, Tanriverdi O. N-Terminal pro-B-Type Natriuretic Peptide Levels are Linked with Modified Child-Pugh Classification in Patients with Nonalcoholic Cirrhosis [NT-ProBNP and Liver Cirrhosis]. Cell Biochem Biophys. 2017, 75(1):111-117.

26. Arozullah AM, Khuri SF, Henderson WG, Daley J. Development and validation of a multifactorial risk index for predicting postoperative pneumonia after major noncardiac surgery. Ann Intern Med. 2001, 135(10):847-57.

27. Jeong HS, Miller TJ, Davis K, Matthew A, Lysikowski J, Lazcano E, Reed G, Kenkel JM. Application of the Caprini risk assessment model in evaluation of non-venous thromboembolism complications in plastic and reconstructive surgery patients. Aesthet Surg J. 2014, 34(1):87-95.

28. Bae MH, Choi WS, Kim KH, Park SH, Kim HW, Lee JH, Lee SW, Yang DH, Park HS, Cho Y, Chae SC, Jun JE. The implications of a fragmented QRS complex and newly reclassified revised cardiac risk index including fragmented QRS in patients undergoing non-cardiac vascular surgery. Int J Cardiol. 2012, 157(2):276-8. 
29. Hackett NJ, De Oliveira GS, Jain UK, Kim JY. ASA class is a reliable independent predictor of medical complications and mortality following surgery. Int J Surg. 2015, 18:184-90.

30. Ciesielska N, Sokołowski R, Mazur E, Podhorecka M, Polak-Szabela A, Kędziora-Kornatowska K. Is the Montreal Cognitive Assessment (MoCA) test better suited than the Mini-Mental State Examination (MMSE) in mild cognitive impairment $(\mathrm{MCl})$ detection among people aged over 60 ? Meta-analysis. Psychiatr Pol. 2016, 50(5):1039-1052.

31. Dunstan DA, Scott N, Todd AK. Screening for anxiety and depression: reassessing the utility of the Zung scales. BMC Psychiatry. 2017,17(1):329.

32. Xie Z, Lv X, Hu Y, Ma W, Xie H, Lin K, Yu X, Wang H. Development and validation of the geriatric depression inventory in Chinese culture. Int Psychogeriatr. 2015, 27(9):1505-11.

33. Dufouil C, Beiser A, McLure LA, Wolf PA, Tzourio C, Howard VJ, Westwood AJ, Himali JJ, Sullivan L, Aparicio HJ, Kelly-Hayes M, Ritchie K, Kase CS, Pikula A, Romero JR, D'Agostino RB, Samieri C, Vasan RS, Chêne G, Howard G, Seshadri S. Revised Framingham Stroke Risk Profile to Reflect Temporal Trends. Circulation. 2017, 135(12):1145-1159.

34. Chen P, Liu Y, Wang Y, Wang A, Zheng H, Zhao X, Yan A, Wang Y. A Validation of the Essen Stroke Risk Score in Outpatients with Ischemic Stroke. J Stroke Cerebrovasc Dis. 2016, 25(9):2189-95.

35. Ellis G1, Spiers M, Coutts S, Fairburn P, McCracken L. Preoperative assessment in the elderly: evaluation of a new clinical service. Scott Med J. 2012, 57(4):212-6.

36. Hamaker ME, Buurman BM, van Munster BC, Kuper IM, Smorenburg CH, de Rooij SE. The value of a comprehensive geriatric assessment for patient care in acutely hospitalized older patients with cancer. Oncologist. 2011, 16(10):1403-12.

37. Kristjansson SR, Nesbakken A, Jordhøy MS, Skovlund E, Audisio RA, Johannessen HO, Bakka A, Wyller TB. Comprehensive geriatric assessment can predict complications in elderly patients after elective surgery for colorectal cancer: a prospective observational cohort study. Crit Rev Oncol Hematol. 2010, 76(3):208-17.

38. Fried LP, Tangen CM, Walston J, et al; Cardiovascular Health Study Collaborative Research Group. Frailty in older adults: evidence for a phenotype. J Gerontol A Biol Sci Med Sci. 2001;56(3): M146M156.

39. Kaiser MJ, Bauer JM, Rämsch C, et al; Mini Nutritional Assessment International Group. Frequency of malnutrition in older adults: a multi- national perspective using the mini nutritional assessment. $J$ Am Geriatr Soc. 2010;58(9):1734-1738.

40. Plassman BL, Langa KM, Fisher GG, et al. Prevalence of cognitive impairment without dementia in the United States. Ann Intern Med. 2008;148(6):427-434.

41. Zhao B, Shang S, Li P, Chen C, Dang L, Jiang Y, Wang J, Huo K, Deng M, Wang J, Qu Q. The genderand age- dependent relationships between serum lipids and cognitive impairment: a cross-sectional study in a rural area of Xi'an, China. Lipids Health Dis. 2019, 18(1):4.

42. Adogwa O, Elsamadicy AA, Vuong VD, Fialkoff J, Cheng J, Karikari IO, Bagley CA. Association between baseline cognitive impairment and postoperative delirium in elderly patients undergoing 
surgery for adult spinal deformity. J Neurosurg Spine. 2018, 28(1):103-108.

43. Makary MA, Segev DL, Pronovost PJ, et al. Frailty as a predictor of surgical outcomes in older patients. J Am Coll Surg. 2010, 210(6): 901-908.

44. Arozullah AM, Daley J, Henderson WG, Khuri SF. Multifactorial risk index for predicting postoperative respiratory failure in men after major noncardiac surgery. The National Veterans Administration Surgical Quality Improvement Program. Ann Surg. 2000;232(2):242-253.

45. Chen T-Y, Anderson DJ, Chopra T, Choi Y, Schmader KE, Kaye KS. Poor functional status is an independent predictor of surgical site infections due to methicillin-resistant Staphylococcus aureus in older adults. J Am Geriatr Soc. 2010;58(3):527-532.

46. Kim S, Brooks AK, Groban L. Preoperative assessment of the older surgical patient: honing in on geriatric syndromes. Clin Interv Aging. 2014, 10:13-27.

47. van Stijn MFM, Korkic-Halilovic I, Bakker MSM, van der Ploeg T, van Leeuwen PAM, Houdijk APJ. Preoperative nutrition status and postoperative outcome in elderly general surgery patients: a systematic review. JPEN J Parenter Enteral Nutr. 2013;37(1):37-43.

48. Kuzu MA, Terzioğlu H, Genç V, et al. Preoperative nutritional risk assessment in predicting postoperative outcome in patients undergoing major surgery. World J Surg. 2006;30(3):378-390.

49. Puvanesarajah V, Jain A, Kebaish K, Shaffrey Cl, Sciubba DM, De la Garza-Ramos R, Khanna AJ, Hassanzadeh H. Poor Nutrition Status and Lumbar Spine Fusion Surgery in the Elderly:

Readmissions, Complications, and Mortality. Spine (Phila Pa 1976). 2017, 42(13):979-983.

50. García-Ramiro S, Cofán F, Esteban PL, Riba J, Gallart X, Oppenheimer F, Campistol JM, Suso S. Total hip arthroplasty in hemodialysis and renal transplant patients. Hip Int, 2008. 18(1): 51-7.

51. Lieu D, Harris IA, Naylor JM, Mittal R. Review article: Total hip replacement in haemodialysis or renal transplant patients. J Orthop Surg (Hong Kong), 2014. 22(3): 393-8.

52. Kildow BJ, Agaba P, Moore BF, Hallows RK, Bolognesi MP, Seyler TM. Postoperative Impact of Diabetes, Chronic Kidney Disease, Hemodialysis, and Renal Transplant After Total Hip Arthroplasty. J Arthroplasty. 2017, 32(9S):S135-S140.

53. Lieberman JR, Fuchs MD, Haas SB, Garvin KL, Goldstock L, Gupta R, Pellicci PM, Salvati EA. Hip arthroplasty in patients with chronic renal failure. J Arthroplasty, 1995, 10(2):191-5.

\section{Table}

Table 3 is available in the Supplemental Files section.

\section{Figures}




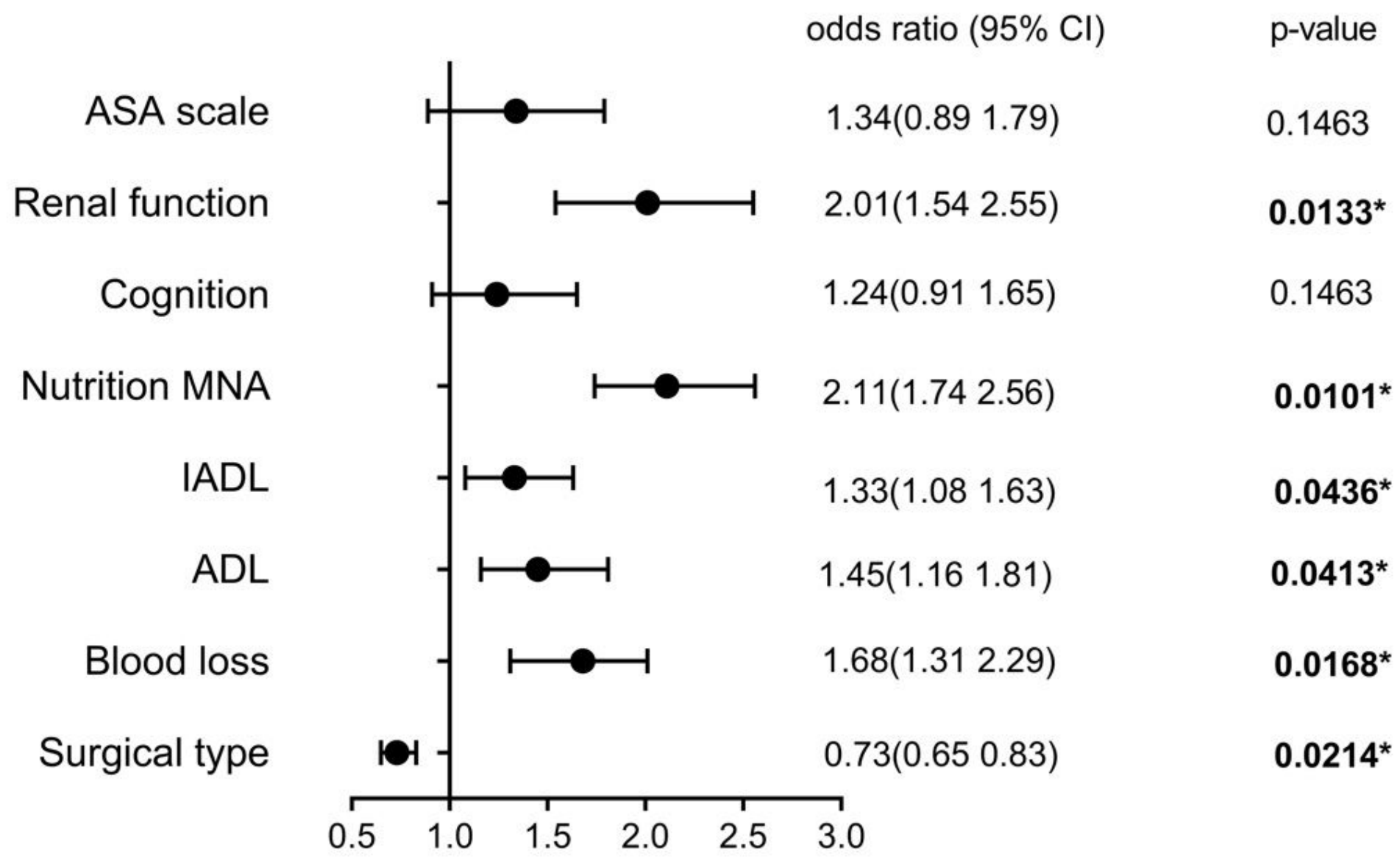

Figure 1

Legend not included with this version.

\section{Supplementary Files}

This is a list of supplementary files associated with this preprint. Click to download.

- Table3.docx

- supplementalfile.docx 